\title{
TRABECULAR BONE TISSUE STRAINS IN THE HEALTHY AND OSTEOPOROTIC HUMAN FEMUR
}

\author{
+*van Rietbergen, B; ***Eckstein, F; ****Koller, B; ****Huiskes, R; *Baaijens, F; **Ruegsegger, $\mathrm{P}$ \\ +*Biomechanics Section, Eindhoven University of Technology, Eindhoven, The Netherlands. P.O. Box 513/5600 MB Eindhoven/The Netherlands, +31 40 2473006, \\ Fax: +31 40 2447355, bertr@wfw.wtb.tue.nl
}

Introduction: A quantitative assessment of bone tissue loading is essential for the understanding of failure mechanisms associated with osteoporosis, implant loosening and cell mediated bone remodeling processes. Trabecular bone microstructural Finite Element ( $\mu \mathrm{FE})$ models generated from microComputed Tomography (CT) scans have recently enabled the calculation of loads in individual trabeculae. Due to restrictions in scanning volume and cputime, however, tissue loads in human bone have only been determined in small test specimens which were loaded in a non-physiological way. For the calculation of physiological tissue loading, natural boundary conditions must be applied, which is possible only when $\mu \mathrm{FE}$ models can represent whole bones. With recently developed micro-CT scanners that can hold larger pieces of bone, and new parallel computers with many gigabytes of memory, this is now possible. The purpose of this study was to determine and compare physiological tissue level strains in a healthy and osteoporotic human proximal femur, using this new equipment and $\mu \mathrm{FE}$ techniques.

Methods: Based on measured BMD values, a healthy and an osteoporotic human femur were selected from a stock of 80 retrieved femurs. The neck BMD value was $122 \%$ of the age-matched average for the healthy femur and $69 \%$ for the osteoporotic one. The femurs were selected such that age, body-weight and length of the female donors were closely matched.

Sequential cross-sectional images of the proximal parts of these femurs were made using a newly developed micro-CT scanner (Scanco $\mu$ CT80) with an 80 $\mathrm{mm}$ field-of-view. 3D voxel models of the bones were generated from the segmented images. Bone voxels were converted to brick elements ${ }^{1}$ of $80 \mu$ in size resulting in $\mu \mathrm{FE}$-models with over 96 million and 71 million elements for the healthy and osteoporotic femur respectively. Elements were assigned a stiffness of $15 \mathrm{GPa}$ for cortical and $10 \mathrm{GPa}$ for trabecular bone tissue. A distributed load of 4-times body-weight (BW) was applied normal to the femoral head surface ${ }^{2}$. The $\mu \mathrm{FE}$-problems were solved using an iterative EBE solver ${ }^{1}$. Thirty processors and 17 GB memory of a SG/Cray Origin2000 computer were used for the calculations. Total cputime was on the order of 20.000 hours per run. Principal strains were calculated for each element and represented in 3-D contour plots. Histograms of the principal strain distribution were calculated for a volume of interest (VOI) that was defined as the largest sphere that would fit in the femoral head.

Results: The strain plots (Fig. 1,2) indicate that compressive tissue strains are found for trabeculae running from the femoral head to the medial cortex, and tensile tissue strains for trabeculae in the perpendicular direction. Strains in the osteoporotic VOI were generally higher than in the healthy VOI and their distribution was wider (Fig. 3). The standard deviation was $77 \%$ of the average for the healthy VOI and $98 \%$ of the average for the osteoporotic VOI indicating that, even when accounting for the higher average, the osteoporotic distribution is wider. For $4 \%$ of the tissue in the osteoporotic VOI strains exceeded 3000 ustrain compared to $0.1 \%$ for the healthy VOI.

Discussion: With the techniques demonstrated here it is now, for the first time, possible to determine physiological tissue level strains in human bones. The results indicate that bone tissue in the healthy situation is more uniformly loaded than in the severe osteoporotic situation and, according to Wolff's law, better adapted to the local loading situation.

Two limitations should be mentioned. First, muscle forces are not included in the model. However, their net effect is included in the joint force and the results thus represent physiological tissue strains in the femoral head and neck. Second, numerical errors and the relatively large $80 \mu$ voxel size can limit the accuracy of the $\mu \mathrm{FE}$ models ${ }^{3}$. However, although local errors in the strains might exist, their effect on histograms and plots will be small ${ }^{1,4}$.

We expect that the methods demonstrated here can offer new information that is of great importance for the understanding of osteoporotic failure and other mechanically induced processes as mentioned in the introduction. Cputime requirements for these analyses, however, are still excessive.

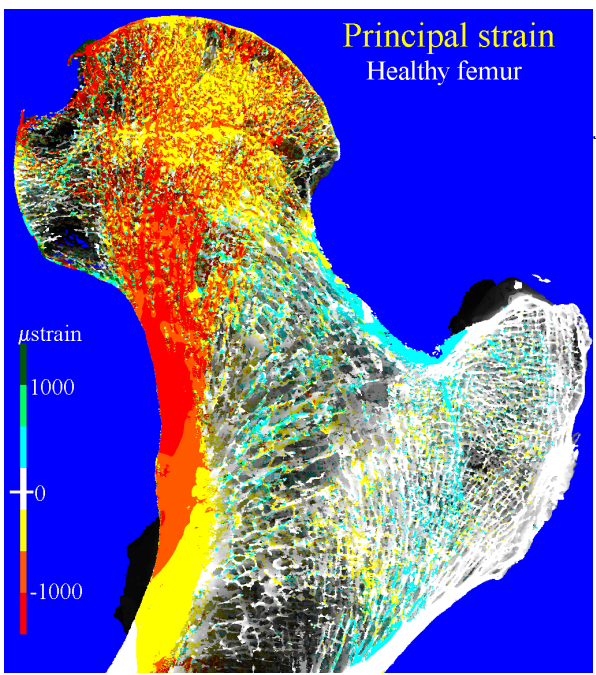

Fig 1 Plot of the $\mu F E$ calculated Principal strain distribution in the healthy femur for a distributed $4 \times B W$ load acting normal to the femoral head. The distal end is fully constrained. The 3-D $\mu F E$ model contains over 96 million 8-node brick elements that are $80 \mu$ in size on all sides. Some of the posterior crosssections are removed to show strains in the trabeculae. Yellow-red: compressive strains, bluegreen: tensile strains.

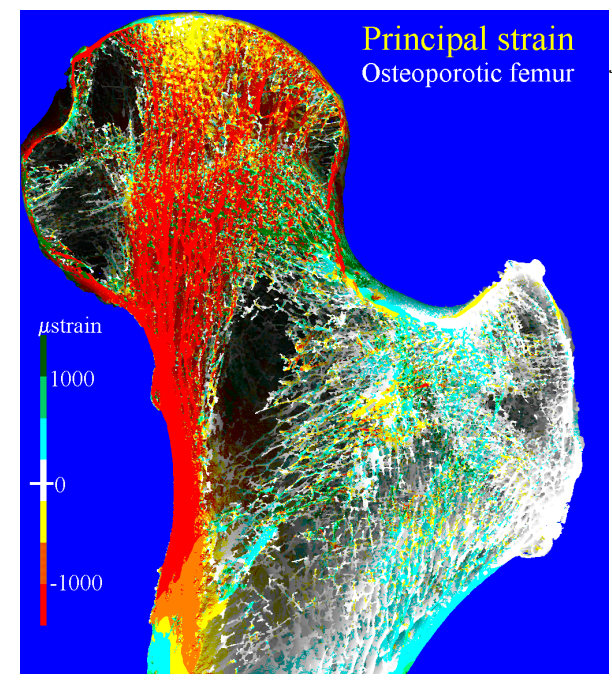

Fig. 2 The same as Fig. 1 for the osteoporotic femur. This $\mu F E$-model contains over 71 million 8-node brick element, also of $80 \mu$ in size on all sides. Note the large empty regions in the femoral head and in the region of Ward's triangle. Also note that in this situation, cortical bone in the neck carries the load that is carried by cortical and trabecular bone together in the healthy situation.

Tissue principal strain in Vol

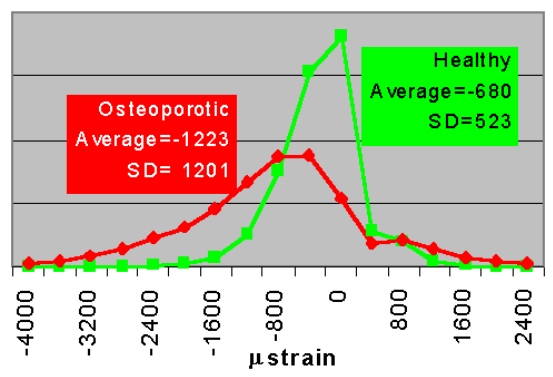

Acknowledgment: this study was supported by an ETH research grant References: 1 van Rietbergen et al., 1995, J. Biomech., 28:69; 2 Bergmann et al., 1993, J. Biomech., 26:969; 3 Ladd and Kinney (1998) $J$. Biomech., 31:941; 4 Ulrich et al., 1998, J. Biomech., 31:1187

**Inst. for Biomedical Eng., ETH and University of Zurich, Switzerland.

***Anatomische Anstalt, University Munchen, Germany.

****Scanco Medical AG, Bassersdorf, Switzerland.

*****Biomechanics Section, University Nijmegen, The Netherlands.
Fig. 3 Distribution of the tissue principal strain in the spherical VOI within the femoral head for the healthy femur (green) and or the osteoporotic femur (red). Average and standard deviation (SD) are indicated in the boxes. 